\title{
A resected case of recurrent ITPN in the remnant pancreas after pancreatoduodenectomy
}

\author{
Kenju Ko ${ }^{1 *}$ D, Yasunori Nishida ${ }^{1}$, Kotaro Sasahara ${ }^{1}$, Hirofumi Kishimoto ${ }^{1}$, Otagiri Noriaki ${ }^{1}$, Katsunori Tauchi ${ }^{1}$, \\ Koji Azuhata ${ }^{2}$ and Kayoko Higuchi ${ }^{3}$
}

\begin{abstract}
Background: Since intraductal tubulopapillary neoplasm (ITPN) is a rare disease, the clinical features of ITPN, especially the characteristics related to recurrence, have not been revealed. We performed a total remnant pancreatectomy for a patient whose ITPN recurred 16 months after pancreatoduodenectomy (PD). We report useful findings to clarify how ITPN reoccurs based on this experience and previously reported cases.

Case presentation: A 61-year-old male patient was diagnosed with pancreatic cancer and underwent PD. However, a postoperative pathologic examination diagnosed ITPN with invasive cancer. After receiving adjuvant chemotherapy, he was hospitalized for pancreatitis 16 months after the operation. He was diagnosed as having recurrence near the pancreato-jejunal anastomosis based on detailed examinations and underwent a remnant total pancreatectomy. From the results of the histopathological examination, he was found to have a recurrence of ITPN as a polypoid mass without invasion distant from the surgical stump of the first operation. Furthermore, tumor cells floating in the main pancreatic duct distant from the main tumor were observed at three locations.

Review of the literature: Including our case, five cases of recurrence in the remnant pancreas after surgery for ITPN have been reported. Recurrence in the main pancreatic duct was observed in four of these five cases. The primary tumor, which recurred in the remnant pancreas after surgery, was characterized as being relatively small and less invasive; however, Ki-67 labeling index was high. In immunohistochemical examination, the expression of MUC6, which is not one of characteristics of ITPN, tended to be positive.

Conclusion: In this case, tumor cells were floating inside the pancreatic duct at several locations. From the results of this case and a review of previous reports, the cause of ITPN recurrence in this case seemed to be due to tumor cells leaving the tumor and implanting into the pancreatic duct.
\end{abstract}

Keywords: Intraductal tubulopapillary neoplasm (ITPN), Recurrence, Remnant pancreas

\section{Background}

According to the current classification of the World Health Organization (WHO) [1], intraductal tubulopapillary neoplasm (ITPN) is defined as a subtype of intraductal neoplasms of the pancreas. The clinical features of ITPN have not been revealed due to its rarity; it is composed of less than $1 \%$ of exocrine pancreas tumors. Therefore, the characteristics related to recurrence are also unknown as

\footnotetext{
* Correspondence: hyunsoo6900@gmail.com

${ }^{1}$ Department of Surgery, Aizawa Hospital, 2-5-1 Honjo, Matsumoto, Nagano

390-8510, Japan

Full list of author information is available at the end of the article
}

there are only a few reports. We experienced a remnant pancreatectomy for a patient whose ITPN recurred 16 months after pancreaticoduodenectomy (PD). We report on useful findings to clarify how ITPN reoccurs based on this case.

\section{Case presentation}

A 61-year-old male patient without a previous medical history was diagnosed with pancreatic cancer in August 2015 and underwent PD. However, a postoperative pathologic examination yielded a diagnosis of ITPN with associated invasive carcinoma. The patient underwent 
routine examinations after receiving postoperative chemotherapy with S-1 for 6 months $(60 \mathrm{mg}$, orally administered twice a day for 28 days followed by a 14-day rest period). During hospitalization due to acute pancreatitis in December 2016, ITPN recurrence was diagnosed by detailed examinations.

Although he used to smoke 20 cigarettes and drink $700 \mathrm{ml}$ of beer a day, the patient quit smoking and drinking after the first surgery. There was no remarkable past history.

\section{First surgery for primary ITPN}

Laboratory data were normal, except for amylase (298 UI/I; normal, 10-20 UI/l) and lipase (352 UI/I; normal, 10-20 UI/ I). Regarding tumor markers, carbohydrate antigen 19-9 (CA19-9) was slightly increased at $37.3 \mathrm{U} / \mathrm{ml}$, but carcinoembryonic antigen (CEA) and DUPAN-2 were within normal limits.

Preoperative computed tomography (CT) showed a tumor with a low-contrast effect approximately $1 \mathrm{~cm}$ in the head of the pancreas and dilatation of the upstream main pancreatic duct (Fig. 1a, b). At the stenosis of the pancreatic duct, there was a tumor that showed a low signal by fat suppression T1WI and a high signal by T2WI and diffusion-weighted imaging (DWI) (Fig. 1c).

Endoscopic retrograde cholangiopancreatography (ERCP) was performed before the operation. ERCP showed an irregular defect in the main pancreatic duct at the head of the pancreas (Fig. 1d). No image suggested mucus in the pancreatic duct. Brush cytology of the stenosis revealed only pancreatic duct epithelial cells with low atypia.

Given that pancreatic cancer was diagnosed based on these examinations, subtotal stomach-preserving pancreatoduodenectomy (SSPPD) was performed in August 2015.

The macroscopic findings of the resected specimen showed that the tumor filled the pancreatic duct (Fig. 2a). A tumor was growing with tubular or cribriform features in the vascular stroma at the main pancreatic duct. The tumor was accompanied by necrosis in some locations and invaded the stroma around the main pancreatic duct (Fig. 2b, c). Mucus production from the tumor was not observed. The results of immunohistochemical staining were as follows: cytokeratin7 (+), cytokeratin19 (+), MUC5AC (-), MUC2 (-), MUC6 (+), chromogranin A $(-)$, synaptophysin (-), and P53 (+). The Ki-67 labeling index was $35.3 \%$, resulting in a final diagnosis of ITPN with associated invasive carcinoma. A histopathological examination revealed no ITPN at the resection stump of the pancreas.

\section{Second surgery for recurrence}

When recurrence was diagnosed, amylase and lipase levels were as high as $269 \mathrm{UI} / \mathrm{I}$ and 784 UI/I, respectively, but the other data were within normal limits. Each
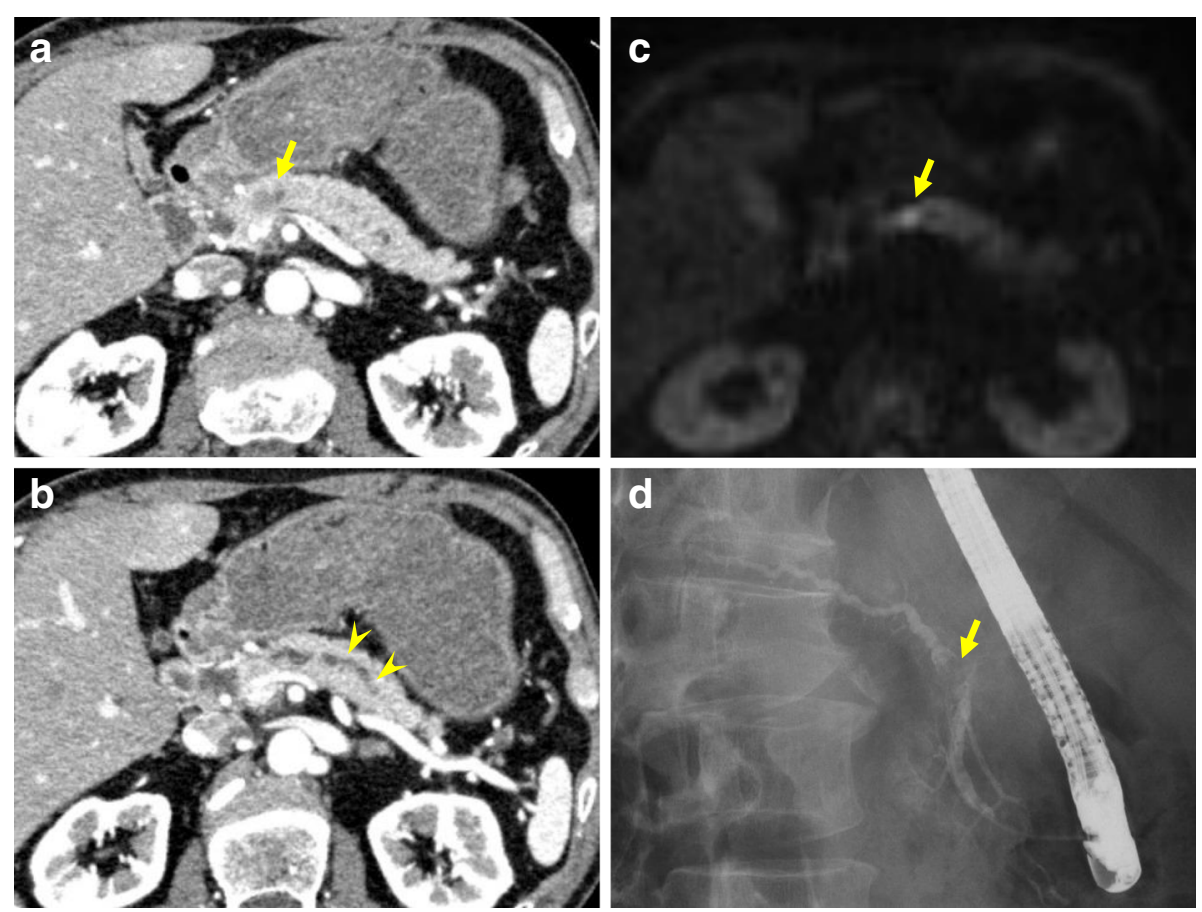

Fig. 1 Preoperative image findings in the first surgery. $\mathbf{a}, \mathbf{b}$ CT showed a low-contrast tumor approximately $1 \mathrm{~cm}$ (arrow) in size and dilatation of the upstream main pancreatic duct (arrowhead). c MRI revealed a tumor showing a high signal by DWI at the stenosis of the pancreatic duct. $\mathbf{d}$ ERCP showed an irregular defect in the main pancreatic duct 


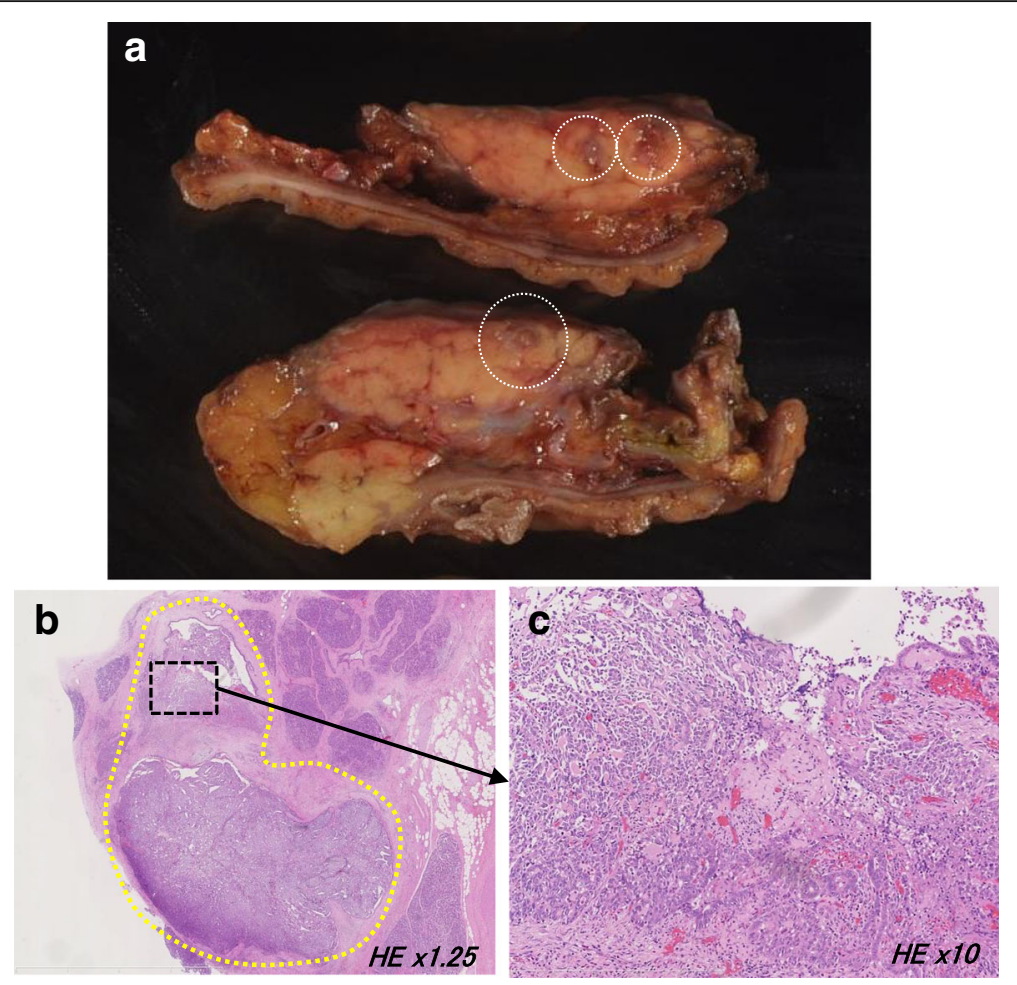

Fig. 2 Macroscopic and histopathological findings of the resected specimen in the first surgery. a Macroscopic findings showed that the tumor filled the pancreatic duct. $\mathbf{b}, \mathbf{c}$ There was a tumor growing with tubular or cribriform features in the vascular stroma at the main pancreatic duct. The tumor was accompanied by necrosis in some locations and invaded the stroma around the main pancreatic duct

tumor marker, such as CEA, CA 19-9, and DUPAN-2, was within normal limits.

The CT showed a low concentration region of $2 \mathrm{~cm}$ in size near the pancreato-jejunal anastomosis, which was similar to the primary ITPN (Fig. 3a). Dilatation of the upstream main pancreatic duct was observed (Fig. 3b). MRI revealed a tumor showing a high signal by DWI at the stenosis of the pancreatic duct (Fig. 3c). Positron emission tomography/computed tomography (PET-CT) revealed an accumulation of SUV max 4.0 at the tumor, while any findings suggestive of other metastases were not observed (Fig. 3d).

As ITPN was not observed at the resection stump of the pancreas by pathological examination of the first surgery, the tumor was diagnosed as recurrence after complete resection rather than residual ITPN in the first operation. A total remnant pancreatectomy was performed in January 2017.

In the histopathological investigation, ITPN polypoid recurrence in the pancreatic duct was observed at a distance of $2.5 \mathrm{~cm}$ from the pancreatic stump. Moreover, three tumor masses were observed in the main pancreatic duct at sites distant from the main tumor (Fig. 4). The main tumor did not show invasion but infiltrated into the main duct and the branches of the pancreatic duct (Fig. 5a, b).
Immunohistochemical staining results revealed cytokeratin7 (+), cytokeratin19 (+), MUC5AC (+/-), MUC2 $(-)$, MUC6 (-), chromogranin A (+/-), synaptophysin $(+/-)$, and p53 $(+)$, which were similar to the primary ITPN. The expression of trypsin, which is indicative of acinar cell carcinoma, was negative. The Ki-67 labeling index was $45.1 \%$, which was very similar to the $35.3 \%$ of the primary lesion. Three tumor masses were observed floating in the main pancreatic duct at sites distant from the tumor, while there were no obvious malignant findings in the pancreatic duct epithelium at each region (Fig. 5c-e).

Recurrence was not observed for 23 months after the second surgery.

\section{Discussion}

ITPN was reported by Yamaguchi et al. [2] as a subclass of the pancreatic ductal tumor and classified separately from intraductal papillary mucinous neoplasm (IPMN) in the 2010 revision of the WHO classification [1]. Although the prognosis of ITPN is better than that of pancreatic cancer $[1,3]$, surgical resection is basic treatment because infiltration and metastasis often occur. Date et al. [4] reported that the prognosis after resection of ITPN was good because the 5-year survival rate was 80.7\%. Furthermore, Date et al. compared cases with and 

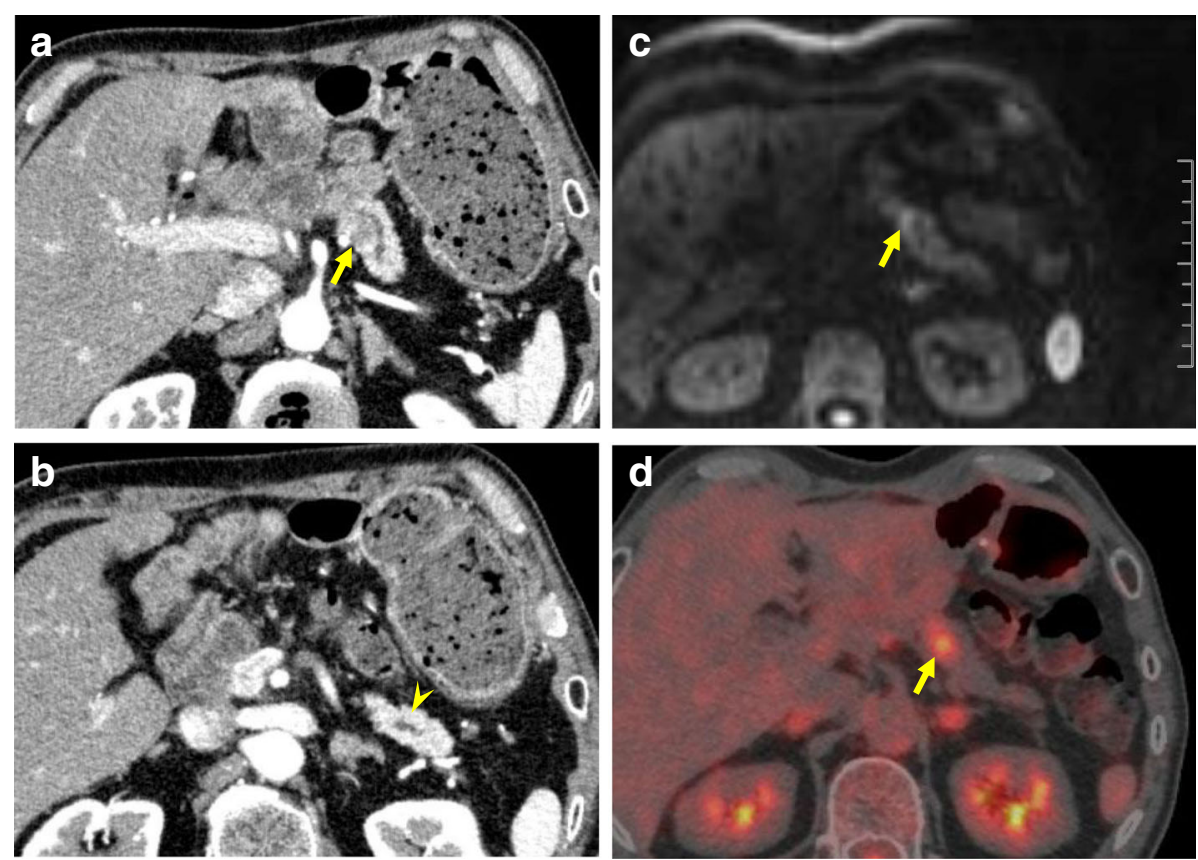

Fig. 3 Preoperative image findings in the second surgery. a CT showed a low concentration region of $2 \mathrm{~cm}$ in size near the pancreato-jejunal anastomosis (arrow). b Dilatation of the upstream main pancreatic duct was observed (arrowhead). c MRI showed a tumor with a high signal by DWI at the stenosis of the pancreatic duct. $\mathbf{d}$ PET-CT revealed the accumulation of SUVmax 4.0 at the tumor

without infiltration and concluded that there was no difference in the 5-year survival rate if the recurrent lesions were completely removed. These results clearly show a better prognosis of resected ITPN compared with invasive pancreatic cancer.

Although the prognosis of ITPN is much better than that of invasive pancreatic cancer, ITPN may reoccur after resection. Following the report by Yamaguchi et al. in 2009, nine cases of detailed descriptions on recurrence after resection have been published, including our case $[2,5-10]$. Interestingly, recurrence in the remnant pancreas was observed in five of nine cases (Table 1). In reviewing these five cases, we noticed that each primary lesion was less invasive and small in size, although the Ki-67 labeling index was high in the pathological diagnosis of primary ITPN. ITPN with infiltration is not rare because the invasion was observed in 54 to $71 \%$ of ITPN cases in previous reports $[4,11,12]$. Less invasion of the primary ITPN may be one of the characteristics of recurrence in the remnant pancreas after surgery.

The primary tumor size may be related to the site of recurrence. In the cases of recurrence in the remnant

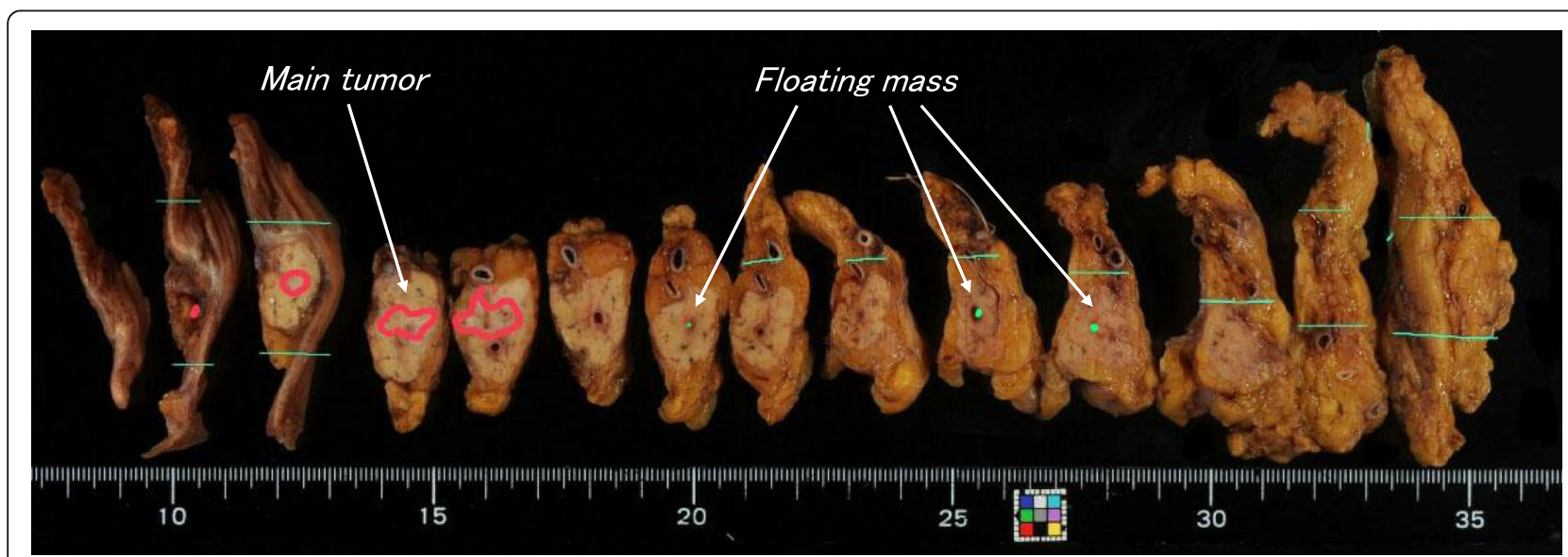

Fig. 4 Resected specimens in the second surgery. Recurrence of ITPN was observed in the pancreatic duct at a distance of $2.5 \mathrm{~cm}$ from the pancreatic stump. Three tumor masses were observed in the main pancreatic duct at sites distant from the main tumor 

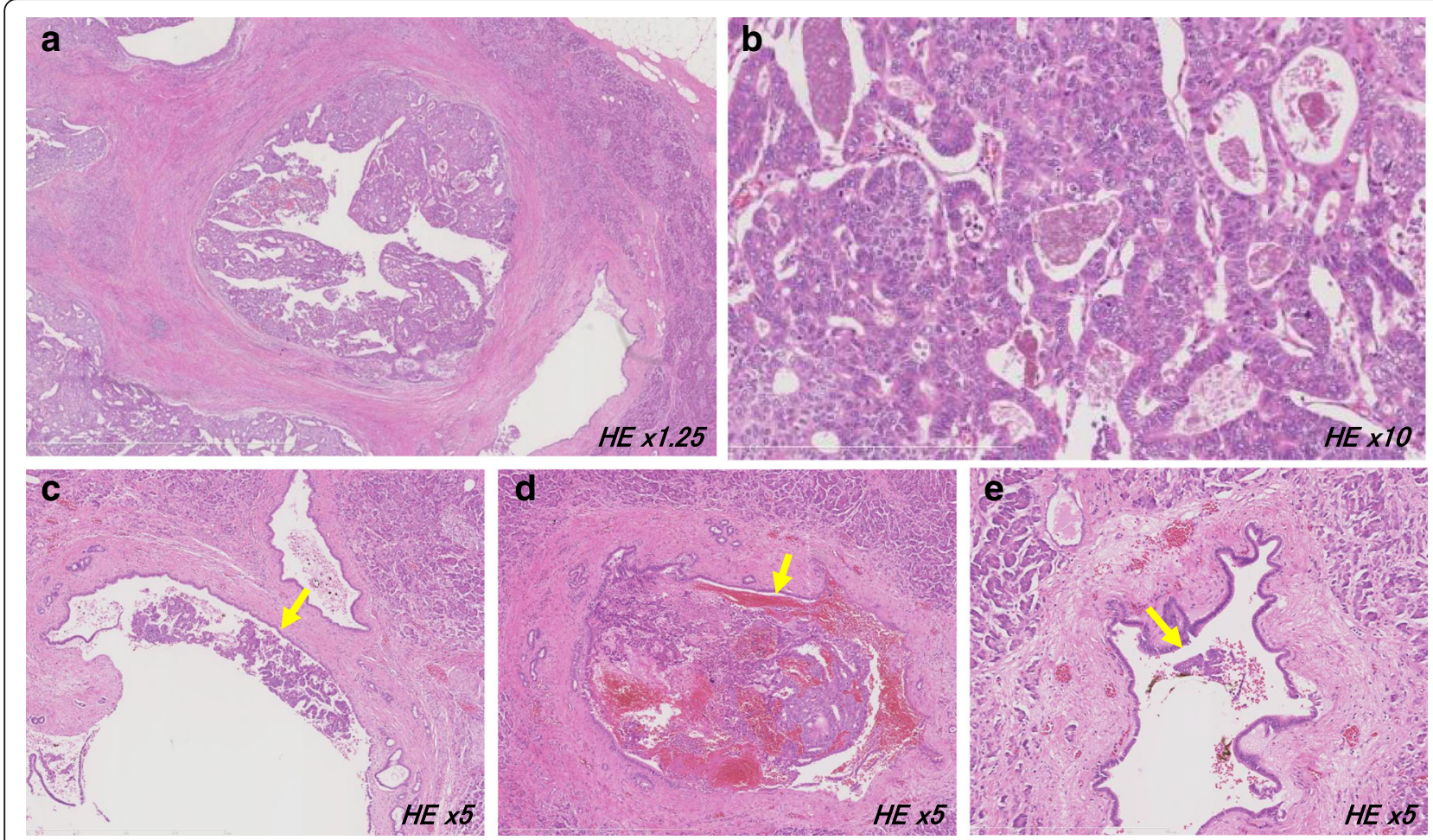

Fig. 5 Histopathological findings of the resected specimens in the second surgery. $\mathbf{a}, \mathbf{b}$ The main tumor infiltrated into the main duct and the surrounding branch of the pancreatic duct. c-e Each tumor mass floated in the main pancreatic duct (arrow). No obvious malignant finding was observed in the pancreatic duct epithelium at each region

pancreas, the primary tumors were less than $20 \mathrm{~mm}$, whereas the primary tumors were greater than $90 \mathrm{~mm}$ in the cases of recurrence in other regions. The characteristics of these five cases indicated that we should focus on the possibility of recurrence in the remnant pancreas during surveillance after surgery even if the primary ITPN was small and less invasive.

The results of immunohistochemical staining were obtained except case 1 (Table 2). The remaining four cases showed a trend of positive staining for CK7 and CK19 and negative staining for MUC2 and MUC5AC as Yamaguchi et al. [2] described as one of the characteristics of ITPN. The expression of MUC6, which was not included in characteristics of ITPN, was positive in three cases. IPMN is classified into four subtypes, including gastric, intestinal, pancreatobiliary, and oncocytic, based on histomorphological features and immunohistochemical features of mucin glycoproteins [13]. The expression of MUC 6 was positive in three types of IPMN subtypes, including gastric type, pancreatobiliary type, and oncocytic type. Furukawa et al. [14] revealed that the differences in subtypes of IPMN were independent predictors of patient prognosis. Basturk et al. [15] described that the expression of MUC6 supports the presence of a pyloropancreatic pathway distinct from the intestinal pathway in IPMN. Even in ITPN, the biological differences of subtypes, such as IPMN, may be related to the mechanism of implantation into the pancreatic duct.

Among these five reports of recurrence in the remnant pancreas, four cases of recurrence were found in the remnant main pancreatic duct, and the remaining case was found in the parenchyma of the pancreas. In addition to the presence of residual tumor after surgery, metastasis through the vessel or implantation into the pancreatic duct may cause the recurrence in the remnant pancreas. Each recurrence was observed in the pancreatic duct, indicating a strong possibility of implantation. In our case, no apparent malignancy was confirmed on the pancreatic duct epithelium, while tumor cells floated in the pancreatic duct. These results suggest that the main recurrent lesion would have been caused by tumor cells leaving the tumor and be implanted in the pancreatic duct epithelium.

Regarding ITPN with multicentric occurrence, Kolby et al. [12] reported a case of total pancreatectomy. Kolby et al. initially performed PD for a pancreas head lesion. However, the operative procedure was changed to total pancreatectomy because malignant cells were found in the pancreatic duct during surgery. After surgery, multiple lesions were histopathologically observed in the body and tail of the resected specimen. Although the case reported by Kolby et al. was not a case of recurrence, it seemed 


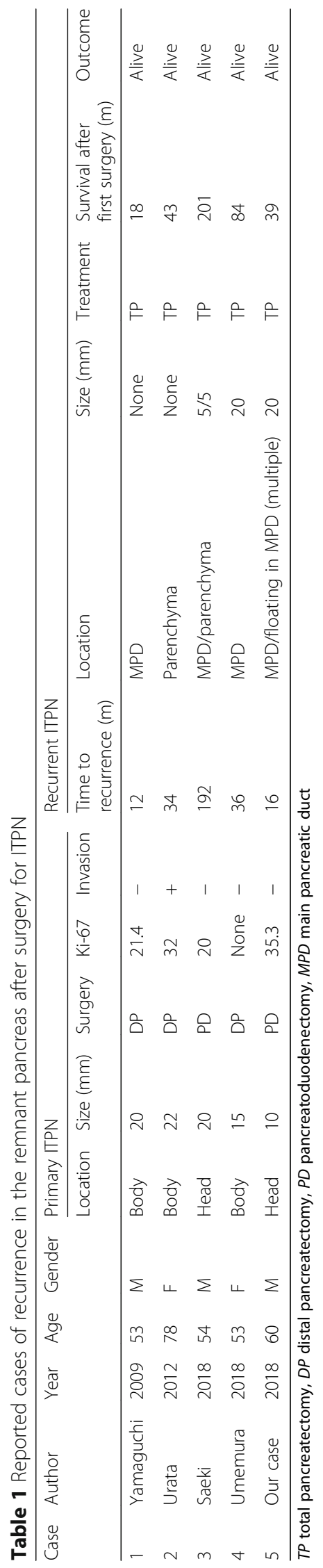


Table 2 Immunohistochemical staining of primary ITPN in five cases that recurred in the remnant pancreas

\begin{tabular}{lllllll}
\hline Case & Author & CK7 & CK19 & MUC2 & MUC6 & MUC5AC \\
\hline 1 & Yamaguchi & None & None & None & None & None \\
2 & Urata & + & + & + & + & + \\
3 & Saeki & + & + & - & + & - \\
4 & Umemura & + & None & - & $+/-$ & - \\
5 & Our case & + & + & - & - & $+/-$ \\
\hline
\end{tabular}

reasonable that implantation was the cause of multiple occurrences because tumor cells floated in the pancreatic duct. Our case exhibited recurrence 16 months after the first surgery, which was different from Kolby et al.'s case. Therefore, we should not forget the possibility that the cause of the main recurrent tumor in our case might be a metachronous lesion. However, images and pathological findings did not offer definite evidence whether the recurrent main tumor was due to implantation or a new lesion. Since the growth rate of the ITPN after implantation is unknown, the period from the first surgery to recurrence is not useful to decide the cause of recurrence.

Implantation may occur in any cancer, but cholangiocarcinoma and lung cancer are diseases in which implantations frequently occur. The commonalities between these tumors and ITPN are currently unknown. As a related study, KAJI et al. [16] reported that interleukin induced by pancreatic cancer enhanced the adhesion of cancer cells to epithelial cells. In addition, Date et al. [17] analyzed a mechanism of recurrence of multifocal main duct IPMN after surgery. Date et al. described the development of a secondary IPMN caused by tumor cells obtaining migratory ability and implanted in the ductal epithelium. A similar process also may occur in ITPN and cause implantation into the pancreatic duct.

To summarize these considerations, the cause of ITPN recurrence in our case seems to be due to tumor cells leaving the tumor and implanting into the pancreatic duct.

\section{Abbreviations \\ CA19-9: Carbohydrate antigen 19-9; CEA: Carcinoembryonic antigen; CT: Computed tomography; DWl: Diffusion-weighted imaging; ERCP: Endoscopic retrograde cholangiopancreatography; HE: Hematoxylin-eosin staining; IPMN: Intraductal papillary mucinous neoplasm; ITPN: Intraductal tubulopapillary neoplasm; MRI: Magnetic resonance imaging; PD: Pancreaticoduodenectomy; PET-CT: Positron emission tomography/computed tomography; SSPPD: Subtotal stomach-preserving pancreatoduodenectomy}

\section{Acknowledgements}

Not applicable

\section{Funding}

Not applicable

\section{Availability of data and materials}

Data sharing not applicable to this article as no datasets were generated or analyzed during the current study. Please contact author for data requests.

\section{Authors' contributions}

KK designed and described the article. YN participated in revising the manuscript. $\mathrm{KS}, \mathrm{HK}$, and $\mathrm{ON}$ advised on the content of the manuscript. KT supervised the editing of the manuscript. KA and KH diagnosed the pathological findings. All authors read and approved the final manuscript.

Ethics approval and consent to participate

Not applicable

Consent for publication

Written informed consent was obtained from the patient for the publication of this case report and any accompanying images.

\section{Competing interests}

The authors declare that they have no competing interests.

\section{Publisher's Note}

Springer Nature remains neutral with regard to jurisdictional claims in published maps and institutional affiliations.

\section{Author details}

'Department of Surgery, Aizawa Hospital, 2-5-1 Honjo, Matsumoto, Nagano 390-8510, Japan. ${ }^{2}$ Department of Pathology, Aizawa Hospital, 2-5-1 Honjo, Matsumoto, Nagano 390-8510, Japan. ${ }^{3}$ Department of Pathology, Okinawa Kyoudo Hospital, 4-10-55 Kohagura, Naha, Okinawa 900-8558, Japan.

Received: 17 December 2018 Accepted: 10 February 2019

Published online: 19 February 2019

\section{References}

1. Adsay NV, Fukushima N, Furukawa T, Hruban RH, Klimstra DS, Klöppel G, et al. Intraductal neoplasm of the pancreas. In: Boston FT, Carneiro F, Hruban $\mathrm{RH}$, Heise ND, editors. World Health Organization classification of 14 tumours of the digestive system. Lyon: IARC Press; 2010. p. 304-13.

2. Yamaguchi H, Shimizu M, Ban S, Koyama I, Hatori T, Fujita I, et al. Intraductal tubulopapillary neoplasms of the pancreas distinct from pancreatic intraepithelial neoplasia and intraductal papillary mucinous neoplasms. Am J Surg Pathol. 2009;33:1164-72

3. Kasugai H, Tajiri T, Takehara Y, Mukai S, Tanaka J, Kudo SE. Intraductal tubulopapillary neoplasms of the pancreas: case report and review of the literature. J Nippon Med Sch. 2013;80:224-9.

4. Date K, Okabayashi T, Shima Y, Iwata J, Sumiyoshi T, Kozuki A, et al. Clinicopathological features and surgical outcomes of intraductal tubulopapillary neoplasm of the pancreas: a systematic review. Langenbeck's Arch Surg. 2016;401:439-47.

5. Bhuva N, Wasan H, Spalding D, Stamp G, Harrison M. Intraductal tubulopapillary neoplasm of the pancreas as a radiation induced malignancy. BMJ Case Rep. 2011. https://doi.org/10.1136/bcr.09.2011.4777.

6. Urata $T$, Naito $Y$, Nagamine M, Izumi $Y$, Tonaki $G$, Iwasaki $H$, et al. Intraductal tubulopapillary neoplasm of the pancreas with somatic BRAF mutation. Clin J Gastroenterol. 2012;5:413-20.

7. Matsuda M, Watanabe G, Hashimoto M, Sasaki K, Tamura T, Imamura T, et al. A case of intraductal tubulopapillary neoplasm of the pancreas with portal vein tumor thrombus. Suizo(in Jpanese). 2014;29:729-34.

8. Matthews Y, McKenzie C, Byrne C, Kench JG. Intraductal tubulopapillary neoplasm of pancreas with associated invasive carcinoma, lymph node, rectal and hepatic metastases. Pathology. 2015;47:169-71.

9. Saeki K, Miyasaka Y, Ohishi Y, Yamamoto T, Matsuda R, Mochidome N, et al. Intrapancreatic recurrence of intraductal tubulopapillary neoplasm (ITPN) 16 years after the initial surgery for noninvasive ITPN: a case report. Surg Case Rep. 2018:4:96.

10. Umemura A, Ishida K, Nitta H, Takahara T, Hasegawa Y, Makabe K, et al. An extremely rare case who underwent total remnant pancreatectomy due to recurrent pancreatic metastasis of intraductal tubulopapillary neoplasm. Clin J Gastroenterol. 2018. https://doi.org/10.1007/s12328-018-0913-x.

11. Basturk O, Adsay V, Askan G, Dhall D, Zamboni G, Shimizu M, et al. Intraductal tubulopapillary neoplasm of the pancreas: a clinicopathologic and immunohistochemical analysis of 33 cases. Am J Surg Pathol. 2017:41:313-25.

12. Kölby D, Thilén J, Andersson R, Sasor A, Ansari D. Multifocal intraductal tubulopapillary neoplasm of the pancreas with total pancreatectomy: report of a case and review of literature. Int J Clin Exp Pathol. 2015;8:9672-80. 
13. Furukawa T, Klöppel G, Volkan Adsay N, Albores-Saavedra J, Fukushima N, Horii A, et al. Classification of types of intraductal papillary-mucinous neoplasm of the pancreas: a consensus study. Virchows Arch. 2005;447:794-9.

14. Furukawa T, Hatori T, Fujita I, Yamamoto M, Kobayashi M, Ohike N, et al. Prognostic relevance of morphological types of intraductal papillary mucinous neoplasms of the pancreas. Gut. 2011;60:509-16.

15. Basturk O, Khayyata S, Klimstra DS, Hruban RH, Zamboni G, Coban I, et al. Preferential expression of MUC6 in oncocytic and pancreatobiliary types of intraductal papillary neoplasms highlights a pyloropancreatic pathway, distinct from the intestinal pathway, in pancreatic carcinogenesis. Am J Surg Pathol. 2010;34:364-70

16. Kaji M, Ishikura H, Kishimoto T, Omi M, Ishizu A, Kimura C, et al. E-selectin expression induced by pancreas-carcinoma-derived interleukin-1 alpha results in enhanced adhesion of pancreas-carcinoma cells to endothelial cells. Int J Cancer. 1995;60:712-7.

17. Date K, Ohtsuka T, Fujimoto T, Tamura K, Kimura H, Matsunaga T, et al. Molecular evidence for monoclonal skip progression in main duct intraductal papillary mucinous neoplasms of the pancreas. Ann Surg. 2017:265:969-77.

\section{Submit your manuscript to a SpringerOpen ${ }^{\circ}$ journal and benefit from:}

- Convenient online submission

- Rigorous peer review

- Open access: articles freely available online

High visibility within the field

- Retaining the copyright to your article

Submit your next manuscript at $\boldsymbol{\nabla}$ springeropen.com 